\title{
FOUR CANDIDATE BINARY STARS WITH STRONG REFLECTION EFFECT FROM THE ZWICKY TRANSIENT FACILITY
}

\author{
BERNHARD, KLAUS ${ }^{\mathbf{1 , 3 , 4}}$ AND HÜMMERICH, STEFAN ${ }^{\mathbf{2 , 3}, \mathbf{4}}$ \\ 1) A-4030 Linz, Austria; e-mail: klaus . bernhard@ liwest . at \\ 2) D-56338 Braubach, Germany; e-mail: ernham@rz-online . de \\ 3) Bundesdeutsche Arbeitsgemeinschaft für Veränderliche Sterne e.V. (BAV), Munsterdamm 90, D-12169 Berlin \\ 4) American Association of Variable Star Observers (AAVSO), 49 Bay State Road, Cambridge, MA 02138, USA
}

\begin{abstract}
We present four stars from the ZTF Catalogue of Periodic Variable Stars whose properties are fully compatible with variable stars of GCVS-type R, that is, short-period binary systems with light variations dominated by strong reflection effect. If verified by spectroscopic studies, our results show that the variability amplitude limit of 1.0 mag $(V)$ currently listed in the International Variable Star Index can be exceeded and may be in need of revision.
\end{abstract}

\section{Introduction}

Under certain conditions, close binary star systems, in particular those with large temperature differences between the components, exhibit what is termed the reflection effect. This phenomenon is caused by radiation of the primary star being re-radiated off (and heating) the corresponding surface parts of the cooler companion (Ruciński, 1969; Samus et al., 2017). Eclipses may or may not be present. In the latter case, the resulting photometric variability is characterized by sinusoidal light curves; the variability period is equal to the orbital period and maximum brightness coincides with the passage of the hot star in front of the companion.

Stars whose variations are dominated by a strong reflection are grouped together under variability type "R" in the General Catalogue of Variable Stars (Samus et al., 2017). The range of light variations is given as $0.5-1.0 \mathrm{mag}(V)$. Such objects are relatively rare and most of them have been little studied. At the time of writing (October 2020), only 137 variables of this type are contained in the International Variable Star Index (VSX) of the AAVSO (Watson et al., 2006), eight of them with variability amplitudes exceeding $1.0 \mathrm{mag}$ $(V)$. Most binary systems with a strong reflection effect have orbital periods of less than 0.5 days.

As has been pointed out, the light curves of R-type variables ${ }^{1}$ are generally sinusoidal but complicated in detail. Besides the re-radiation of the primary's light and the heating of the irradiated surface of the companion by absorbed light, there occurs a subsequent

\footnotetext{
${ }^{1}$ Here, and throughout the paper, the term 'R-type variables' is used to refer to variable stars of GCVS-type R. It is not to be confused with stars of spectral type R, which are also often photometric variables.
} 
redistribution of heat over the entire surface of the cooler star (e.g. Budaj, 2010), which influences the observed light curve pattern. For this reason, detailed study of the reflection effect also helps to understand the properties of the outer stellar layers.

\section{Observations and analysis}

The Zwicky Transient Facility (ZTF) is a sky survey situated at the Palomar Observatory. It takes up the legacy of its predecessor, the Palomar Transient Factory, and has been observing the entire available northern sky since 2017. The ZTF camera employs e2v CCD231-C6 devices and is mounted on the Palomar 48-inch Samuel Oschin Schmidt Telescope. Highquality data in two passbands ( $g$ and $r$ ) are collected every night down to a limiting magnitude of $r \sim 20.6$ mag and made publicly available (Bellm et al., 2019a; Bellm et al., 2019b; Masci et al., 2019).

Obviously, ZTF data are well suited for the study of variable stars. Chen et al. (2020) exploited ZTF Data Release 2 and compiled a catalogue of 781602 periodic variable stars (The Zwicky Transient Facility Catalog of Periodic Variable Stars). ${ }^{2}$ We systematically searched this catalogue for candidate R-type variables by examing the light curves of an extended sample of stars in detail. Since the catalog does not contain a category for R-type variables, it can be assumed that any such objects have been assigned to other categories of short-period variables, such as RR Lyr or W UMa stars. While these types of variable stars have much smaller variability amplitudes than R-type variables, no upper limits for variability amplitudes were enforced (cf. the classification criteria listed in Chen et al., 2020).

Therefore, the light curves of all objects with a period shorter than 0.5 days $(\mathrm{P}<0.5 \mathrm{~d})$ and an amplitude of $\operatorname{amp}(r)>1.0$ mag were investigated. 2030 stars matched the selection criteria. To narrow down the number of candidates, objects with strongly discrepant amplitudes or periods in $r$ and $g$ were discarded in order to ensure high-quality light curves in both passbands. The light curves of the remaining 1167 objects, which we hereafter refer to as the final sample, were then visually checked.

In accordance with the classifications derived by Chen et al. (2020), the final sample mostly consists of eclipsing binaries with deep primary eclipses and RR Lyrae stars of type RRab, which exhibit the typical asymmetric light curves and amplitudes that are significantly larger in $g$ (Fig. 1).

\footnotetext{
${ }^{2}$ http://variables.cn:88/
} 


\section{ZTFJ173604.93-015458.8}

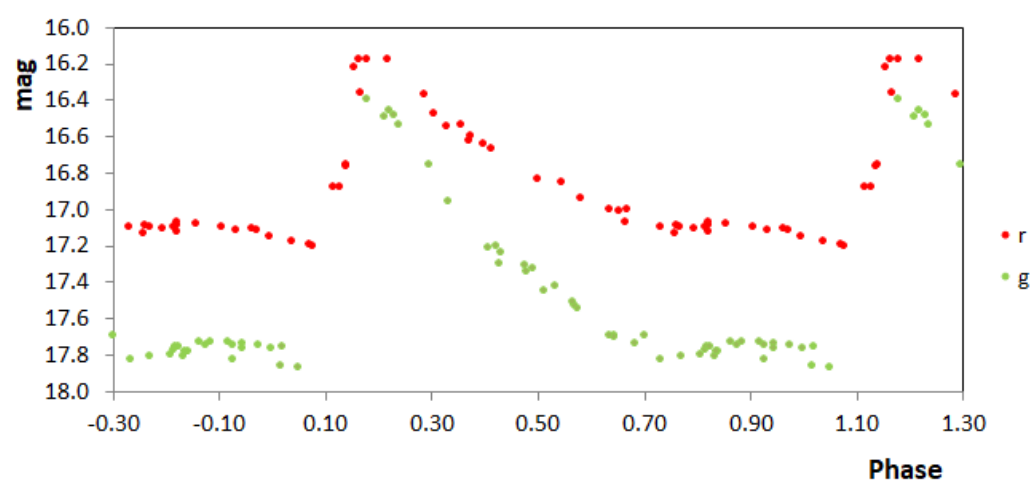

Fig.1 Example phase plot of an RRab star (ZTFJ173604.93-015458.8; P = 0.4362979 d).

Another significant part of final sample was made up of cataclysmic variables, which, however, can generally be identified by increased scatter in the phase plot (Fig. 2), which is due to superposed irregular variations. All these objects were discarded.

ZTFJ170053.31+400357.6

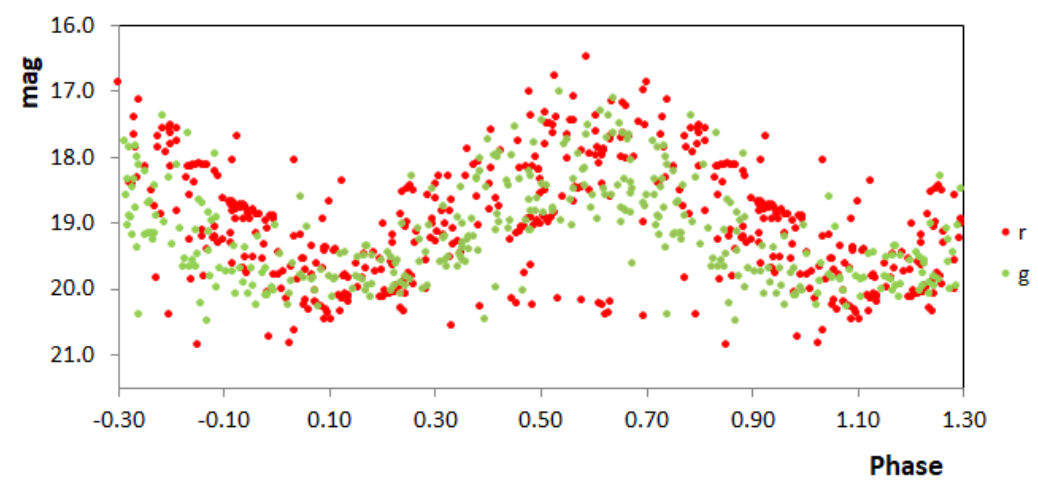

Fig.2 Example phase plot of a cataclysmic variable $($ ZTFJ170053.31+400357.6 = V1237 Her; $\mathrm{P}=0.080869 \mathrm{~d}$; type AM Her).

Particular attention was paid to objects with similar amplitudes in both passbands or objects with $\operatorname{amp}(r)>\operatorname{amp}(g)$. These are properties typical of R-type variables that can be used to differentiate them from other classes of variable stars such as pulsators, which are usually characterized by $\operatorname{amp}(g)>\operatorname{amp}(r)$.

The periods from Chen et al. (2020) of the remaining R-type candidates were checked and, if necessary, refined using the ANOVA algorithm as implemented in the programme package PERANSO (Paunzen \& Vanmunster, 2016). Apart from the fact that some of the period values had to be halved, the agreement between the periods given by Chen et al. (2020) and our period solutions was very good, with the differences amounting to less than $0.00001 \mathrm{~d}$ in all cases. 


\section{Results}

In addition to four stars with properties fully compatible with R-type variables, we recovered the two known R-type variable NQ Cyg (ZTFJ194954.56+380823.0) and the central star of the planetary nebula Ou 5 (ZTFJ211420.03+434136.0; Corradi et al., 2014). This shows that the above mentioned methodology is in principle suited for the search for stars with strong reflection effect. NQ Cyg and ZTFJ211420.03+434136.0 exhibit the typical features of Rtype stars - near sinusoidal and monoperiodic variations on a short period (Fig. 3). The PanSTARRS image of the sky region around ZTFJ211420.03+434136.0 retrieved via ALADIN is presented in Fig. 4.

The observed variability amplitude of NQ Cyg is approximately the same in both passbands $(\operatorname{amp}(r) \sim \operatorname{amp}(g))$, while $\operatorname{amp}(\mathrm{r})>\operatorname{amp}(\mathrm{g})$ in the case of ZTFJ211420.03+434136.0. This behaviour is in line with other known R-type variables, which show increasing amplitudes from the B- to the I band, as for example in the case of KV Vel (e.g. Hilditch et al, 1996).

Phase plots of the other four stars, which we here present as very good R-type variable star candidates, are illustrated in Fig. 5. The corresponding ephemerides as well as other essential data are listed in Table 1. All stars exhibit (near-)sinusoidal light curves and amplitudes that are either similar in both passbands or follow $\operatorname{amp}(r)>\operatorname{amp}(g)$.

Table 1: Objects with strong reflection effect in the ZTF Catalogue: The columns denote: (1) ZTF ID; an asterisk denotes a known R-type variable; (2) RA (J2000; GAIA DR2); (3) DEC (J2000; GAIA DR2); (4) $G$ mag; (5) period (d), as calculated with PERANSO; (6) amplitude in the $g$ band; (7) amplitude in the $r$ band; (8) epoch from Chen et al. (2020).

\begin{tabular}{|c|c|c|c|c|c|c|c|}
\hline ID & $\mathrm{RA}[\mathrm{h} \mathrm{m} \mathrm{s}]$ & $\mathrm{DEC}\left[{ }^{\circ} \cdots\right]$ & $\mathrm{G}[\mathrm{mag}]$ & period $[\mathrm{d}]$ & $\begin{array}{l}\text { Amp g } \\
{[\mathrm{mag}]}\end{array}$ & $\begin{array}{l}\text { Amp r } \\
{[\mathrm{mag}]}\end{array}$ & $\begin{array}{l}\text { Epoch } \\
\text { [Min, HJD] }\end{array}$ \\
\hline ZTFJ020634.68+563518.2 & 020634.682 & +563518.17 & 18.8221 & $0.203675(2)$ & 1.01 & 1.30 & $2458368.935(2)$ \\
\hline ZTFJ030429.27+472115.3 & 030429.270 & +472115.20 & 18.0331 & $0.202613(2)$ & 0.74 & 1.15 & $2458480.807(2)$ \\
\hline ZTFJ060646.19+310000.9 & 060646.192 & +310000.84 & 18.7639 & $0.168913(2)$ & 1.01 & 1.13 & $2458511.699(2)$ \\
\hline ZTFJ190048.52--105815.1 & 190048.522 & -105815.16 & 19.0082 & $0.184712(15)$ & 1.62 & 2.60 & $2458345.829(2)$ \\
\hline ZTFJ194954.56+380823.0* & 194954.575 & +380823.16 & 16.3716 & $0.311590(2)$ & 1.52 & 1.53 & $2458650.970(3)$ \\
\hline ZTFJ211420.03+434136.0* & 211420.035 & +434136.04 & 18.6043 & $0.364225(2)$ & 1.06 & 1.26 & $2458219.027(4)$ \\
\hline
\end{tabular}

Strong reflection effect with an amplitude in excess of one magnitude is only expected in systems consisting of stars of very different temperatures. Under certain circumstances, white dwarf stars may qualify as the hot component, which is consistent with the classification of ZTFJ211420.03+434136.0 as primary star of a planetary nebula and the spectral type of $\mathrm{K}$ that Weidmann et al. (2020) list for the cool component. 
ZTFJ194954.56+380823.0

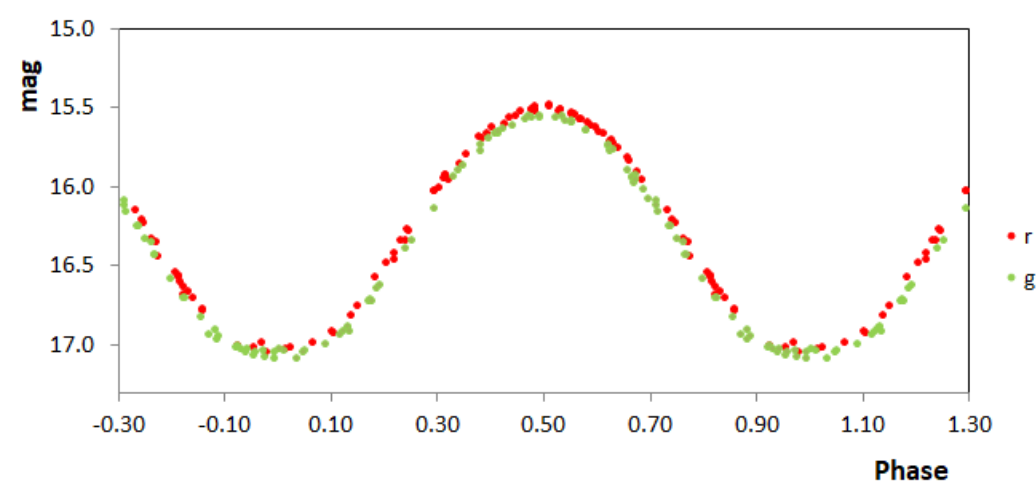

ZTFJ211420.03+434136.0

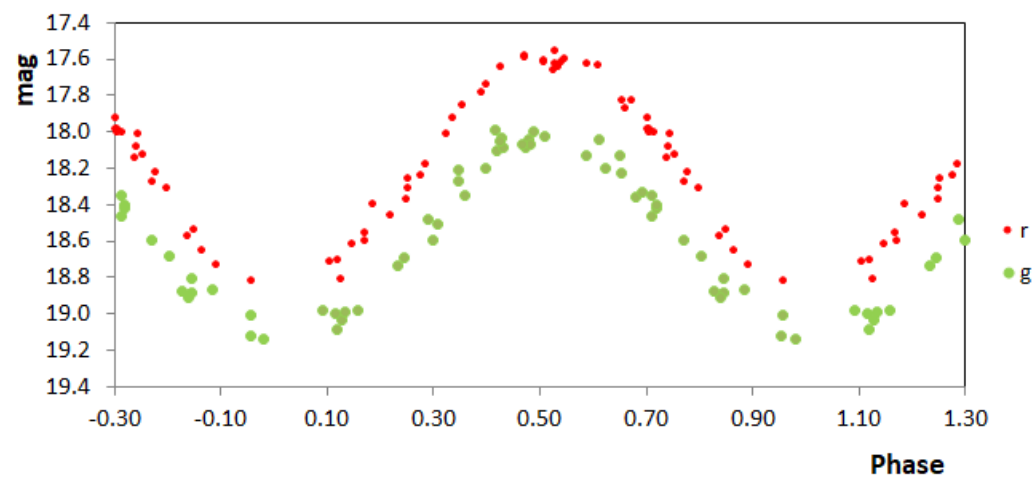

Fig.3 Phase plot of the known R-type variables NQ Cyg (ZTFJ194954.56+380823.0; $P=$ $0.311590 \mathrm{~d}$ ) and the central star of the planetary nebula Ou 5 (ZTFJ211420.03+434136.0; $P=$ $0.364225 \mathrm{~d}$ ) which were recovered using the described methodology.

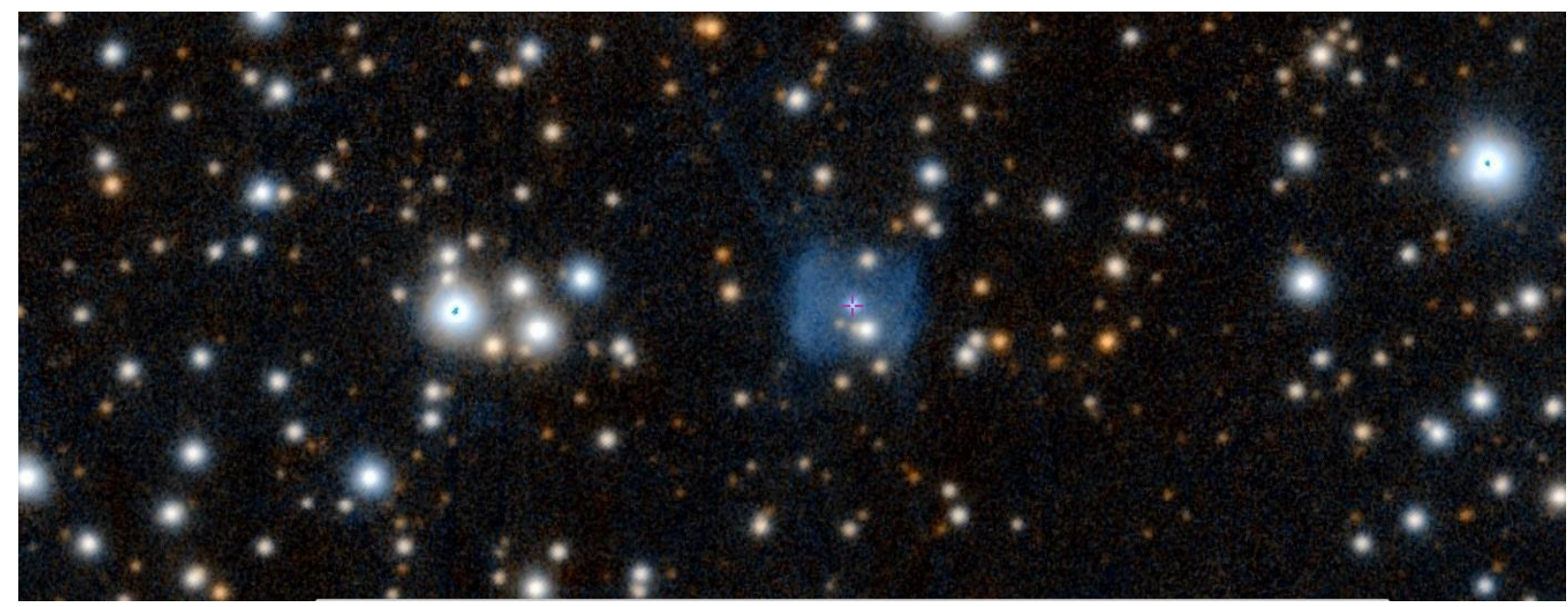

Fig.4 Pan-STARRS DR1 colour frame of the planetary nebula Ou 5, whose central star is ZTFJ211420.03+434136.0 (field of view: $3.6 \times 1.3$ arcmin). 


\section{ZTFJ020634.68+563518.2}

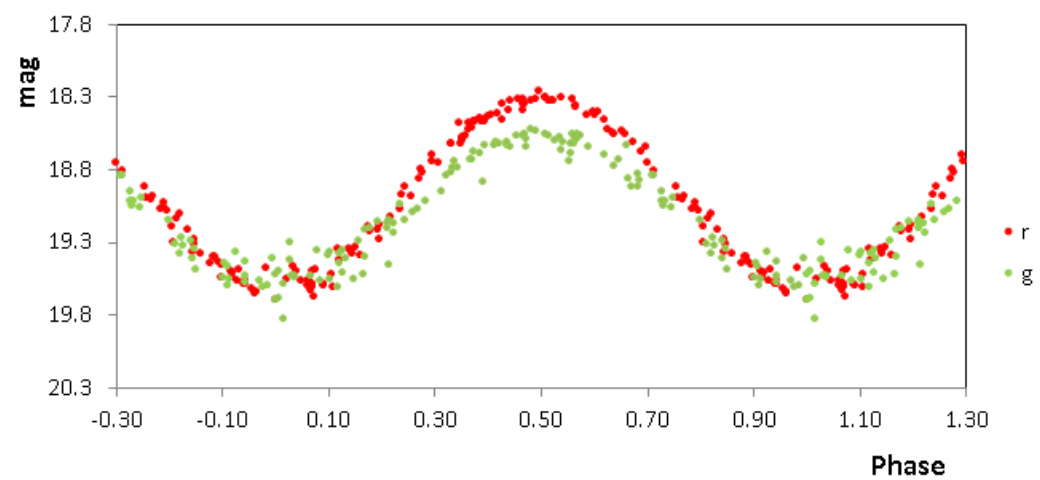

ZTFJ030429.27+472115.3

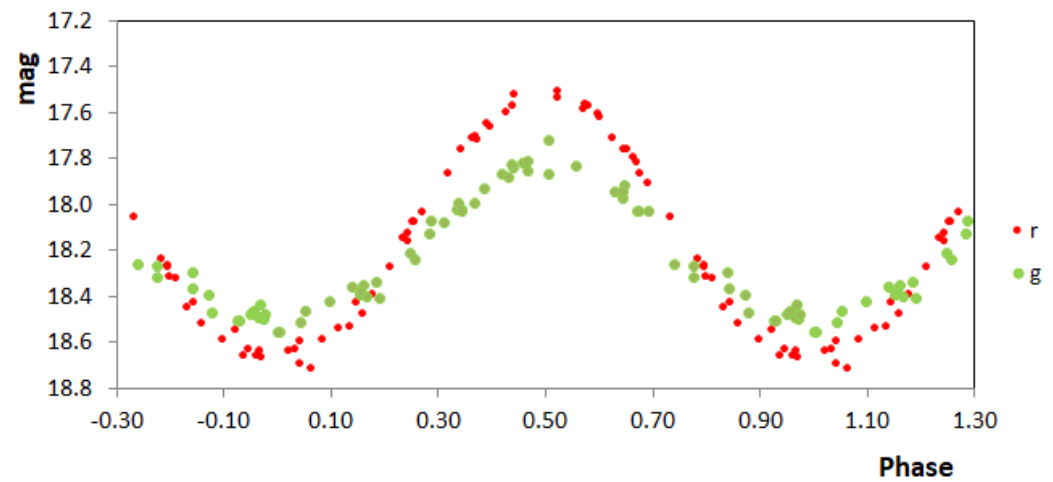

ZTFJ060646.19+310000.9

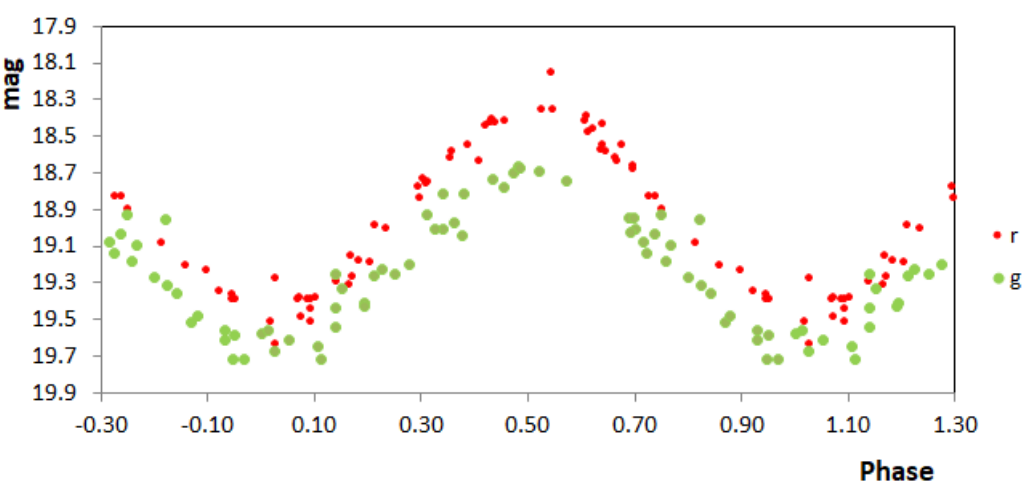

Fig.5 Phase plots of the newly identified R-type variable star candidates (from top to bottom) ZTFJ020634.68+563518.2 $(P=0.203675 \mathrm{~d})$; ZTFJ030429.27+472115.3 $(P=0.202613 \mathrm{~d})$ and ZTFJ060646.19+310000.9 $(P=0.168913 \mathrm{~d})$; 


\section{ZTFJ190048.52-105815.1}

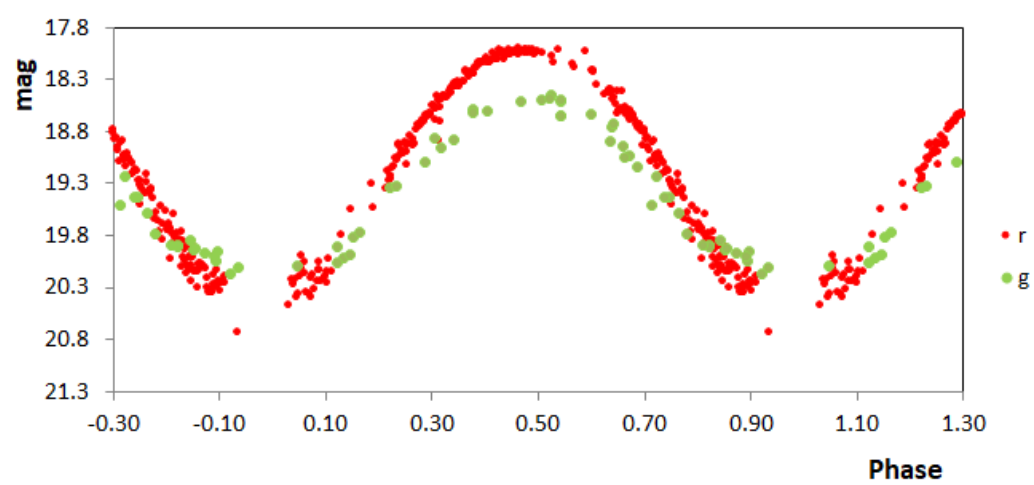

Fig.5 (continued) Phase plot of the newly identified R-type variable ZTFJ190048.52$105815.1(P=0.184712 \mathrm{~d})$.

The void of data points in the folded light curve of ZTFJ211420.03+434136.0 (Fig. 3, bottom panel) near phase $\varphi=0$ corresponds to a deep eclipse, which has been documented by Corradi et al. (2014). Interestingly, a similar phenomenon is present in the light curve of ZTFJ190048.52-105815.1 (Fig. 5, bottom panel). The expected minimum magnitude of the sinusoidal R-type variability is well within the magnitude range covered by the ZTF survey (20.8 mag and 20.6 mag in the $g$ and $r$ bands, respectively; cf. Bellm et al., 2019a) and we see no ready explanation for the observed gaps in the light curve. A search for eclipses in this object, therefore, seems promising.

\section{Conclusion}

The present work presents four variable stars from the Chen et al. (2020) catalogue, whose properties are fully compatible with R-type variables, that is, short-period binary systems with light variations dominated by strong reflection effect. These objects should be confirmed by spectroscopic studies. If proven to be indeed R-type variables, we suggest to increase the listed maximum amplitudes of this kind of variable stars to $1.62(\mathrm{~g})$ and $2.60(\mathrm{r})$, as evidenced by ZTFJ190048.52-105815.1, which is a particularly interesting target for further study.

\section{Acknowledgements:}

We thank the anonymous referee for his comments that helped to improve the paper. This paper made use of data from the VizieR catalogue access tool, the interactive sky atlas ALADIN, both operated at CDS, Strasbourg (France), and of the International Variable Star Index (VSX) database, operated at AAVSO, Cambridge, Massachusetts (USA). 


\section{References}

Bellm, E.C., Kulkarni, S. R., Graham, M. J.et al., 2019, PASP, 131, 018002

https://ui.adsabs.harvard.edu/abs/2019PASP..131a8002B

Bellm, E. C., Kulkarni, S. R., Barlow, T. et al. 2019, PASP, 131, 068003

https://ui.adsabs.harvard.edu/abs/2019PASP..131f8003B

Budaj, J., 2011, AJ, 141, 59

https://ui.adsabs.harvard.edu/abs/2011AJ....141...59B

Chen, X., Wang, S., Deng, L., de Grijs, R., Yang, M., Tian, H., 2020, ApJS, 249, 18 https://ui.adsabs.harvard.edu/abs/2020ApJS..249...18C

Corradi, R. L. M., Rodríguez-Gil, P., Jones, D. et al., 2014, MNRAS, 441, 2799

https://ui.adsabs.harvard.edu/abs/2014MNRAS.441.2799C

Hilditch, R. W., Harries, T. J., Hill, G., 1996, MNRAS,.279,.1380

https://ui.adsabs.harvard.edu/abs/1996MNRAS.279.1380H

Masci, F. J., Laher, R. R., Rusholme, B. et al., 2019, PASP, 131, 018003

https://ui.adsabs.harvard.edu/abs/2019PASP..131a8003M/

Paunzen, E., \& Vanmunster, T. 2016, Astron. Nachr., 337, 239

https://ui.adsabs.harvard.edu/abs/2016AN....337..239P

Ruciński, S. M., 1969, Acta Astronomica, 19, 245

https://ui.adsabs.harvard.edu/abs/1969AcA....19..245R/

Samus, N.N., Kazarovets, E.V., Durlevich, O.V., Kireeva, N.N., Pastukhova, E.N., General Catalogue of Variable Stars: Version GCVS 5.1, 2017, Arep, 61, 80

https://ui.adsabs.harvard.edu/abs/2017ARep...61...80S/

Watson, C. L., Henden, A. A., Price, A., 2006, Society for Astronomical Sciences Annual Symposium, 25, 47

https://ui.adsabs.harvard.edu/abs/2006SASS...25...47W

Weidmann, W. A., Mari, M. B., Schmidt, E. O. et al., 2020, A\&A,. 640A,.10

https://ui.adsabs.harvard.edu/abs/2020A\%26A...640A..10W 